\title{
Is congenital toxoplasmosis still an important clinical problem?
}

\author{
Ali Joman Alghamdi ${ }^{1}$, Ali Alabbas ${ }^{1,2}$, Monika Tadla ${ }^{1}$, Catherine Agyemfra-Kumi ${ }^{1,3}$, \\ Agata Tarkowska', Wanda Furmaga-Jabłońska' \\ ${ }^{1}$ Medical University of Lublin, Poland \\ ${ }^{2}$ Umm Al-Qura university, Makkah, Kingdom of Saudi Arabia \\ ${ }^{3}$ Adventist Hansdale Hospital, Hindsale Illinois, USA
} Alghamdi AJ, Alabbas A, Tadla M, Agyemfra-Kumi C, Tarkowska A, Furmaga-Jabłońska W. Is congenital toxoplasmosis still an important
clinical problem? J Pre-Clin Clin Res. 2017; 11(2): 167-170. doi: 10.26444/jpccr/81167

\begin{abstract}
Introduction. Congenital toxoplasmosis is one of the most common intrauterine inherited diseases. The risk and severity of infection depends on the time of transmission during pregnancy. The earlier the infection, the lower the risk of transmission but with serious estimated outcomes and vice versa.

Case report. A newborn with congenital toxoplasmosis was born to a mother who was seronegative at the beginning of pregnancy, and was not aware of toxoplasmosis risk factors during pregnancy. The newborn was born with features of small for gestational age and with intracranial calcification revealed in cranial ultrasonography, which emphasized the need for further investigations. Neonatal serological panel and cerebrospinal fluid analysis were both confirmative of congenital toxoplasmosis. Ophthalmological examination showed the typical whitish focus of retinochoroiditis which later led to a blindness in one eye. Additionally, hydrocephalus was progressing slowly until it became stable in 5 months.

Objective. The aim of this study was to present the case of congenital toxoplasmosis in order to underline the lack of social awareness concerning risk factors in pregnant women. Thus, educational campaigns against the consumption of raw meat and unpasteurized milk while pregnant may prevent the recurrence of such cases. Moreover, proper washing of fruits and vegetables are advised in this respect.
\end{abstract}

Key words

Toxoplasma gondii, congenital toxoplasmosis, risk factors, prophylaxy, undercooked meat, Good hygiene, Spiromycin

\section{INTRODUCTION}

Toxoplasmosis is a wide-spreading infection caused by an obligate intracellular parasite called Toxoplasma gondii (T. gondii) [1]. People usually become infected by two main routes: foodborne (eating raw or undercooked meat, especially lamb or pork, drinking non-pasteurized milk) or zoonotic (ingestion of oocytes from infected cat's faeces: accidentally while cleaning a cat's litter box, eating unwashed vegetables or fruits growing in contaminated soil, drinking contaminated water. $[2,3]$. Additionally, a vertical transmission (from mother to foetus) is a third possible way, as noted by Wolf et al. in 1939 [4, 5]. Recently, congenital toxoplasmosis (CT) has become a medical issue of concern with an annual incidence of about 190,000 cases worldwide [4]. However, the incidence rate varies accordingly to environmental and hygienic factors besides eating habits $[3,6]$. In Poland, a recent study found the frequency of CT to be around $0.05-0.1 \%$ of live births [3]. The neonates showed no clinical symptoms at birth in most of the cases, except for a $10 \%$ minority with classical signs of CT: chorioretinitis, hydrocephalus and intracranial calcifications $[7,8]$. The present report describes an interesting case of a neonate with symptomatic CT who was born to mother who was seronegative at the beginning of pregnancy.

Address for correspondence: Ali Joman Alghamdi, Medical University of Lublin, Leszczynskigo 15A/9, 20069 Lublin, Poland

E-mail:ali_joman_2@hotmail.com

Received: 7 November 2017; accepted: 12 December 2017

\section{CASE REPORT}

A 22-year-old mother pregnant with her third child presented to the outpatient clinic for a routine prenatal visit in her first trimester and was screened for Toxoplasmosis. It should be noted that screening for T. gondii in this mother while pregnant was performed only once. Toxoplasma gondii specific antibodies were negative at the time. According to her medical history, she had eaten raw meat while pregnant. According to her, she had no idea she should desist from eating raw meat while pregnant.

The female newborn was born at 38 weeks of the gestational period, in a good general state of health (she was scored 9 points according to Apgar score). However, she was small for the gestational age: birth weight $-2510 \mathrm{~g}$ ( 3 rd percentile); head circumference $-31 \mathrm{~cm}(<3$ rd percentile). Due to these features, an intracranial ultrasonography (USG) was performed, which showed calcifications in the cerebral cortex. Subsequently, expanded diagnostics tests for nonbacterial infections were performed because of the suspicion of a congenital infection which, however, revealed negative results for Cytomegalovirus (CMV), but with a high titer of T. gondii Immunoglobulin M (IgM) and Immunoglobulin G (IgG). After a confirmatory test revealed toxoplasmosis, the 8-day-old newborn was transferred to the Department of Infant Pathology at the University Children's Hospital in Lublin, eastern Poland. On admission, the infant was in a good condition; physical examination showed no abnormalities except for a slight enlargement of the anterior fontanelle $(3.5 \times 2.5 \mathrm{~cm})$. Serum tests of T. gondii antibodies were performed for the second time, and positive results of elevated $\mathrm{T}$. gondii antibodies 
level were reported - IgM 3.01 Index (positive > 1.0 Index), IgG $>700.00 \mathrm{IU} / \mathrm{ml}$ (positive $>10.0 \mathrm{IU} / \mathrm{ml}$ ). The mother was then tested post-delivery for $\mathrm{T}$. gondii and showed positive results; IgM $2.33(\mathrm{~N}<0.55)$, IgG $287 \mathrm{IU} / \mathrm{ml}(\mathrm{N}<4 \mathrm{IU} / \mathrm{ml})$.

A diagnostic lumbar puncture was performed and cerebrospinal fluid (CSF) collected for analysis. Cerebrospinal fluid (CSF) showed a slightly yellowish-cloudy discoloration which appeared clear after centrifugation. Cell count of 57 cells/ $\mu \mathrm{l}$ with the following distribution: $5 \%$ granulocytes, 94\% lymphocytes, $1 \%$ monocytes and numerous erythrocytes. A positive Pandy's reaction with a protein level of $169 \mathrm{mg} / \mathrm{dl}$ and glucose level of $40 \mathrm{mg} / \mathrm{dl}$ was also observed. Most importantly, T. gondii DNA was detected in CSF by polymerase chain reaction (PCR), which confirmed the diagnosis of CT.

Intracranial USG was again performed on the newborn and revealed 3 periventricular calcifications in the left hemisphere, and 2 calcifications in right hemisphere frontal and parietal lobes with diameter of approximately 4- $7 \mathrm{~mm}$. Abdominal USG was also performed but showed no abnormalities. Chest X-ray showed an inflammatory density in her right pulmonary hilum, suggesting congenital pneumonia.

The infant was consulted by a team of specialists, including ophthalmologists, cardiologists, neurologists and otolaryngologists. Results of her eye examination revealed a whitish focus of retinochoroiditis (which is a typical feature for ocular toxoplasmosis) in both eyes, measuring 2-disc diameter in size. Echocardiography revealed a patent foramen ovale and first degree tricuspid insufficiency with regurgitation jet up to $2 \mathrm{~m} / \mathrm{s}$. However, neurological and audiometric examinations showed no abnormalities at that time.

As a result, she was started on a treatment course of Pyrimethamine plus Sulfadiazine and folinic acid. Iron supplement was also given when the neonate developed anaemia. After about 5 weeks, the infant was discharged in a stable condition with a weight gain $(3,634 \mathrm{~g}-3 \mathrm{rd}$ percentile $)$ and head circumference of $36.5 \mathrm{~cm}$ ( $<3 \mathrm{rd}$ percentile). At discharge, the child was in a good general state, the USG evaluation of the brain revealed only few calcifications with no other abnormalities.

The patient was set to complete a 12-months course of treatment at home with scheduled follow up visits. After 2 weeks, the infant was brought to the Neurology Outpatient Clinic for a control intracranial USG, which revealed a developing hydrocephalus with an Evans ratio of 0.36 . This progressed slowly until the infant was 5 months olds and reached an Evans ratio of 0.42; from then on, the hydrocephalus became stable.

Today, the girl is 2 and half year's old and regularly visits a neurologist and ophthalmologist for continuous evaluation. She is cheerful, reacting to commands with a slightly decreased muscle tone. However, she is blind in one eye with poorly developed speech and suffers from sleeping disturbances.

\section{DISCUSSION}

Background. Toxoplasmosis is a common parasitic infection caused by $\mathrm{T}$. gondii which is transmitted by cat faeces carrying parasitic oocysts $[6,9]$. Once food or water is contaminated with the oocysts, it becomes a potential source of infection whenever it is consumed by humans and other mammals. Raw meat or unpasteurized milk of an infected animal is another common route to acquire $\mathrm{T}$. gondii infection $[1,2,6]$. Additionally, in pregnancy, maternal infection poses a high risk of a vertical transmission through the placenta leading to a spectrum of potential complications $[6,10]$. The risk of transmission increases remarkably as pregnancy proceeds with an estimated risk of $15 \%, 44 \%$ and $71 \%$ in the 1 st, 2 nd and 3rd trimesters, respectively [4]. This could be explained by the increased permeability of placental barriers throughout the pregnancy. On the contrary, the earlier the infection, the worst the outcomes are to be expected, for instance, it can lead to miscarriages or serious neurological defects $[2$, $6,11]$. In severe to moderate cases, the classical triad of CT is occasionally seen at birth, including; retinochoroiditis, intracranial calcifications and hydrocephalus in $80 \%, 40 \%$ and $20 \%$, respectively [12]. Other signs and symptoms may include congenital cataract, pneumonitis, encephalitis, hepatosplenomegaly and lymphadenopathy, among others [12]. In severe cases, infection with T. gondii may increases the risk of miscarriage [2]; however, about $90 \%$ of neonates with CT are asymptomatic at birth but still at risk of later development of clinical symptoms, mostly ophthalmologically-related [2, 13]. It is worth noting that this case did not present the classical triad of CT at birth; however, she developed it later during her first year of life.

Screening. To alleviate the severity of symptoms, an early detection of T. gondii infection followed by proper treatment are crucial. Therefore, screening programmes should be promoted to a wide range of social groups, esspecially those at high risk. In particular, HIV positive patients, immunosuppressed mothers, and those with abnormal results of foetal ultrasound evaluation [13]. Ideally, women should be screened for T. gondii before planning pregnancy. In the case of non-planned pregnancy, a screening test should be performed as soon as pregnancy is confirmed. If the test reveals a negative result, then repeated tests should be made at least once per trimester throughout the pregnancy [3]. Despite the huge advantages behind these programmes, several problems stand in the way of running them on a global basis, for example, variation topographical prevalence, test availability, uncertainty of intrauterine treatment efficacy, and elevated financial concerns [13]. Population unawareness of the possible risks may lead to the omission or delay of screening which, in turn, poses another challenge. Taking into account the maternal immunological status and the serological test results, the foetus poses a transmission risk of different degrees. The foetus is at risk if the mother shows an acute $\mathrm{T}$. gondii infection $(\mathrm{IgM}+/ \mathrm{IgG}+)$ or chronic infection (IgM-/IgG+) while immunocompromised. According to Pomares \& Montoya, in the case of an immunocompetent mother who presents no prior infection with IgM-/IgG-, the foetus is theoretically at no risk of CT; however, as shown in the presented case, the mother is at high risk of developing a primary infection during the pregnancy [4]. Therefore, this cannot be definitely ruled out in the presented case.

Diagnosis. T. gondii infection can be diagnosed directly by detecting the parasitic DNA via PCR, or indirectly based on the serological findings of specific antibodies [3]. Either method can be applied at different stages of pregnancy with 
variable sensitivity and specificity results. Maternal diagnosis of toxoplasmosis is mainly confirmed by the serological finding of T. gondii antibodies $\operatorname{Ig}$ M, $\operatorname{IgA}, \operatorname{IgE}$ and $\operatorname{IgG}[4,6]$. A positive maternal result poses a risk for the foetus to develop CT. In order to confirm the prenatal infection, detection of parasitic DNA in the foetal amniotic fluid is a method of choice [4]. Similarly, postnatal serological observation of IgM in the newborn or increased titer of IgG for the following 12 months are indicative of CT [7]. It is noteworthy that alternative tools which are proposed in routinely medical practice, such as USG, may raise the suspicion of CT. USG findings, including intracranial calcification, hydrocephalus or intrauterine growth restriction, are suggestive of congenital disease but not specific for toxoplasmosis since many etiologies share the same symptoms [4].

Serological test. Detection of T. gondii specific antibodies is an important, indirect tool for establishing a diagnosis of toxoplasmosis, and to identify whether the infection has an acute or a chronic term [3]. Therefore, several techniques were introduced in the laboratory to obtain higher specificity and sensitivity results. Examples include immunofluorescence antibody test (IFAT), immunosorbent agglutination assay (ISAGA), enzyme-linked immunosorbent assay (ELISA) and enzyme-linked immunofiltration assay (ELIFA) [13, 14]. There is also the Immunoblotting technique (western blot) which, in comparison to others, is the most reliable for detecting antibodies following electrophoresis of denatured toxoplasmic antigen $[10,13,14]$. Following a recent infection, an increased level of IgM is noticed within 5 days to weeks, reaching a peak in $1-2$ months, before dropping gradually. Whereas a rise in IgG level is usually observed about 2 weeks after the infection, with a peak between $3-6$ months, and plays the major immunological role after IgM subsides [13]. Based on the above, possible maternal serological findings and their interpretation are shown in Table $1[3,13]$.

However, it must always be borne in mind that an IgM positive reading might be misleading since they may last for an extended period of time. Hence, an IgG avidity test is very useful for estimating the duration of infection [10]. In other words, a high IgG avidity over $30 \%$ is generally suggestive of an infection older than 4 months [3]. Another issue of concern is the capability of IgG antibodies to cross placenta in prenatal period, leading to false positive results in foetal serological panel postnatally. Therefore, a comparative IgG profile between mother and child (CGMC test) is the assay of choice to differentiate between maternal and foetal antibodies and has a sensitivity of $\sim 82.9 \%$ and specificity of $~ 93 \%$ [7]. Lastly, a new noninvasive technique had been introduced for the diagnosis and follow-up of CT in infants, based on the detection of specific anti toxoplasmosis IgG in saliva [4]. Table 1 shows possible maternal serological findings and their interpretation [3]

Molecular testing. Detection of the T. gondii's genetic material by PCR is a precise and direct diagnostic tool that can be applied prenatally and postnatally $[3,4]$. A body fluid sample is required for that purpose. In the case of prenatal diagnosis, the collection of an amniotic fluid sample not earlier than week 18 is optimal $[3,13]$, whereas a postnatal sample collection of cerebrospinal fluid (CSF), blood or urine is sufficient to run PCR [4]. Initially, a series of laboratory procedures is performed in order to extract
Table 1.

\begin{tabular}{|c|c|c|c|}
\hline $\lg M$ & $\lg G$ & Interpretation & Recommendation \\
\hline \multirow[t]{3}{*}{+} & + & $\begin{array}{l}\text { Most likely a recent infection with } \\
\text { high }\end{array}$ & $\begin{array}{l}\text { Start prophylactic with } \\
\text { spiromycin. Then }\end{array}$ \\
\hline & & possibility of foetal transmission. & $\begin{array}{l}\text { shift to pyrimethamine and } \\
\text { sulfadiazine }\end{array}$ \\
\hline & & & If foetal infection is confirmed \\
\hline \multirow[t]{3}{*}{+} & - & $\begin{array}{l}\text { Possibly false positive results or a } \\
\text { recent }\end{array}$ & $\begin{array}{l}\text { Repeat test in } 2-3 \text { weeks } \\
\text { looking for lgG }\end{array}$ \\
\hline & & $\begin{array}{l}\text { infection, take into consideration } \\
\text { a foetal }\end{array}$ & titer elevation. \\
\hline & & transmission potential risk & \\
\hline- & + & Possibly a chronic infection $>1$ year & Repeat test in 2- 3 weeks \\
\hline \multirow[t]{2}{*}{-} & - & No infection or extremely recent & Repeat test each trimester \\
\hline & & Infection & \\
\hline
\end{tabular}

DNA material from the obtained sample. Then, a targeted gene, including P30 gene, TGR1E, ribosomal DNA or B1, is amplified. The final product of the amplification is analyzed in electrophoresis gel [8]. Subsequently, a positive result of PCR is confirmation of the CT diagnosis, since they have a high sensitivity of up to $97.4 \%$ and $100 \%$ specificity [12].

Treatment in pregnancy. The main goal of treatment is primarily to prevent foetal infection, and if detected, to reduce its severity if the latter was not effective [13]. If the mother is infected prior to week 18 of gestation, foetal prophylactic treatment with spiromycin is indicated [4]. Spiromycin is a macrolide antibiotic that cannot cross, but rather accumulate in the placenta, thereby preventing vertical transmission of T. gondii [13].

After week 18, amniocentesis is safely preformed to assess foetal condition [3]. A negative foetal result indicates the necessity for continuing the spiromycin treatment until the end of pregnancy [13]. In contrast, a confirmed foetal infection implies an immediate change in the treatment plan to pyrimethamine and sulfadiazine $[4,13]$, alongside folinic acid, which is an integral part of the regimen to compensate the consequent side-effects of pyrimethamine. Moreover, clindamycin, azithromycin or clarithromycin may substitute sulfadiazine in the case of allergy [4]. However, it is worth mentioning that pyrimethamine and sulfadiazine are contraindicated during the first trimester due to their potential teratogenic side-effects $[4,12,13]$.

Prevention. To prevent T. gondii infection, screening and education programmes for women prior to and during pregnancy should be publicly promoted $[6,13]$. Screening enables early detection and better prognosis. Similarly, educational programmes will create awareness of the 'do's and don'ts' during pregnancy, especially - as in the presented case - the eating of raw meat, unwashed fruits or vegetables, and drinking unpasteurized milk, which should be avoided when pregnant. The avoidance of handling cat litter while pregnant also prevents coming into contact with the T. gondii cysts. A randomized survey was performed in 2006 in the USA which included 1,200 obstetricians/ gynecologists, and revealed that the average health practitioner informed pregnant patients about the risks involved in handling raw 
meat (67\%) and consuming undercooked meat (78\%), and all of them informed about the risks involved in handling cat litters. It is recommended that meat should be cooked at a temperature higher than $62 \mathrm{C}, 70 \mathrm{C}$, and $73 \mathrm{C}$ for a whole cut meat, ground meat, and all poultry, respectively. Also, all meat should be kept at a very low temperature (-20C) for at least 48 hours in order to kill T. Gondii cysts in the tissues [15]. Furthermore, good hygiene is necessary, especially the washing of hands after contact with meat or soil, as well as the proper washing of fruits and vegetables [3]. This, in turn, will decrease infection rates during pregnancy and promote safer pregnancies for mothers and unborn babies, and especially for those living in the countryside who are at higher risk due to their daily activities and lifestyle [6].

\section{CONCLUSIONS}

$\mathrm{CT}$ is among the most common congenital infections worldwide. Although asymptomatic presentation at birth is most commonly observed, serious complication may develop later. However, health education prior pregnancy plays a major role in preventing the infection. Women at childbearing age should all be made aware of the potential risk factors of acquiring and then transmitting infection. Consumption of raw meat and unwashed vegetables, as well as contact with cats faeces during pregnancy are the main risk factors. Once they are infected, an early detection and treatment grant a better outcome.

\section{REFERENCES}

1. Mattos C, Spegiorin L, Meira C, Silva T, Ferreira A, Nakashima F, et al. Anti-Toxoplasma gondii antibodies in pregnant women and their newborn infants in the region of São José do Rio Preto, São Paulo, Brazil. Sao Paulo Medical Journal 2011; 129(4): 261-266.

2.Radoń-Pokracka M, Piasecki M, Lachowska A, Baczkowski S, Spaczyńska J, Górecka M, et al. Assessment of the implementation of the infectious diseases screening programmes among pregnant women in the Lesser Poland region and comparison with similar programmes conducted in other European Union countries. Ginekologia Polska 2017; 88(3): 151-155.

3. Milewska-Bobula B, Lipka B, Golas E, Debski R, Marczynska M, Paul $\mathrm{M}$, et al. Recommended management of toxoplasma gondi infection in pregnant women and their children. Przegl Epidemiol. 2015; 69(2): 291-298.

4. Pomares C, Montoya JG. Laboratory Diagnosis of Congenital Toxoplasmosis. Journal of Clinical Microbiology 2016; 54(10): 2448-2454.

5. Wolf A, Cowen D, Paige B. Human toxoplasmosis: occurrence in infants as an encephalomyelitis verification by transmission to animals. Science 1939; 89: 226-227.

6. Salamon D, Bulanda M. Toxoplasma gondii and women of reproductive age: an analysis of data from the Chair of Microbiology, Jagiellonian University Medical College in Cracow1. Annals of Parasitology 2014; 60(4): 291-296. Retrieved August 28, 4014.

7. Gross U, Lüder CGK, Hendgen V, Heeg C, Sauer I, Weidner A, et al. Comparative Immunoglobulin G Antibody Profiles between Mother and Child (CGMC Test) for Early Diagnosis of Congenital Toxoplasmosis. Journal of Clinical Microbiology 2000; 38(10): 3619-3622.

8. Vidigal PVT, Santos DVV, Castro FC, Couto JC, Vitor RW, Brasileiro Filho G. Prenatal toxoplasmosis diagnosis from amniotic fluid by PCR. Revista da Sociedade Brasileira de Medicina Tropical 2002; 35(1): 1-6.

9. Naghili B, Abbasalizadeh S, Tabrizi S, Rajaii M, Akramiyan M, Alikhan $\mathrm{H}$, et al. Comparison of IIF, ELISA and IgG avidity tests for the detection of anti-Toxoplasma antibodies in single serum sample from pregnant women. Le Infezioni in Medicina 2017; (1): 50-56.

10. Stajner T, Bobic B, Klun I, Nikolic A, Srbljanovic J, Uzelac A, et al. Prenatal and Early Postnatal Diagnosis of Congenital Toxoplasmosis in a Setting With No Systematic Screening in Pregnancy. Medicine 2016; 95(9).

11. Nowakowska, D, Colón I, Remington JS, Grigg M, Golab E, Wilczynski J, Sibley LD. Genotyping of Toxoplasma gondii by Multiplex PCR and Peptide- Based Serological Testing of Samples from Infants in Poland Diagnosed with Congenital Toxoplasmosis. Journal of Clinical Microbiology 2006; 44(4): 1382-1389.

12. Modrzejewska M, Patalan J, Kulik U, Czeszyńska M. Ocular manifestation of congenital toxoplasmosis, clinical implication - case report. Polish Gynaecology 2016; 87(03).

13. Paquet C, Yudin MH, Allen VM, Bouchard C, Boucher M, Caddy S, et al. Toxoplasmosis in Pregnancy: Prevention, Screening, and Treatment. Journal of Obstetrics and Gynaecology Canada 2013; 35(1): 78-79.

14. Chumpitazi BF, Boussaid A, Pelloux H, Racinet C, Bost M, GoullierFleuret A. Diagnosis of congenital toxoplasmosis by immunoblotting and relationship with other methods. Journal of Clinical Microbiology 1995; 33(6): 1479-1485.

15. Maldonado Y, Read J. Diagnosis, Treatment, and Prevention of Congenital Toxoplasm osis in the United States. Pediatrics 2017; 139(2): e20163860. 